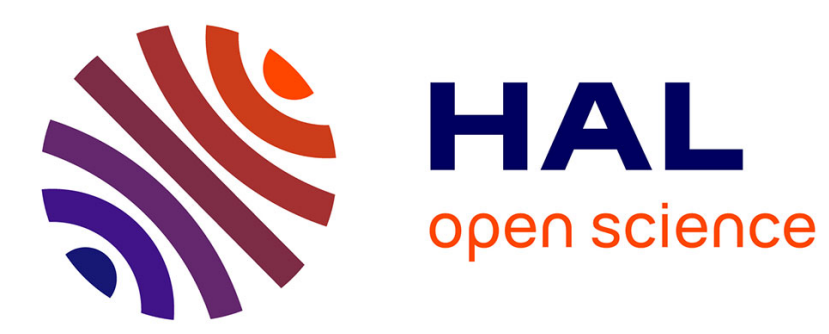

\title{
Optimized Single Carrier Transceiver for Future Sub-TeraHertz Applications
}

Simon Bicaïs, Jean-Baptiste Doré, Gregory Gougeon, Yoann Corre

\section{To cite this version:}

Simon Bicaïs, Jean-Baptiste Doré, Gregory Gougeon, Yoann Corre. Optimized Single Carrier Transceiver for Future Sub-TeraHertz Applications. 2020 45th International Conference on Acoustics, Speech, and Signal Processing (ICASSP), May 2020, Barcelona, Spain. hal-02470916

\section{HAL Id: hal-02470916 https://hal.science/hal-02470916}

Submitted on 7 Feb 2020

HAL is a multi-disciplinary open access archive for the deposit and dissemination of scientific research documents, whether they are published or not. The documents may come from teaching and research institutions in France or abroad, or from public or private research centers.
L'archive ouverte pluridisciplinaire HAL, est destinée au dépôt et à la diffusion de documents scientifiques de niveau recherche, publiés ou non, émanant des établissements d'enseignement et de recherche français ou étrangers, des laboratoires publics ou privés. 


\title{
Optimized Single Carrier Transceiver for Future Sub-TeraHertz Applications
}

\author{
Simon Bicaïs ${ }^{1}$, Jean-Baptiste Doré ${ }^{1}$, Grégory Gougeon ${ }^{2}$, Yoann Corre ${ }^{2}$ \\ ${ }^{1}$ CEA-Leti, Minatec Campus, Grenoble, France \\ ${ }^{2}$ Siradel, Saint-Grégoire, France \\ contact: simon.bicais@cea.fr; ycorre@siradel.com
}

\begin{abstract}
The performance of sub-THz communications, contemplated for the next generation of wireless networks, are significantly degraded by oscillator phase noise. In this paper, we address the design of a single carrier transceiver resilient to phase noise. This problem is treated in two steps: First, we derive for phase noise channels the optimum symbol detection criterion. Second, we propose a phase noise robust modulation scheme with a comprehensive and efficient structure. System level simulations modeling the sub-THz propagation channel of a rich outdoor environment through ray tracing are also presented. Results show that optimizing the modulation and demodulation schemes is necessary to achieve high spectral efficiency in the presence of phase noise.
\end{abstract}

Index Terms-Sub-TeraHertz communications, Ray-tracing, Phase noise, Digital modulation, Design optimization.

\section{INTRODUCTION}

With an important amount of unused spectrum, the subTeraHertz (sub-THz) frequencies between 90 and $300 \mathrm{GHz}$ are contemplated to realize high-data rate wireless communications and hence to fulfill the requirements of the nextgeneration of wireless networks [1]. Several beyond 5G applications are considered for sub-THz systems [2]: high capacity backhaul, enhanced hot-spot kiosk and also short-range communications. Despite the evolution of semi-conductor technology, the design of new physical layer algorithms is required to achieve high data-rate sub-THz communications. Traditional coherent schemes do not consider the specific radio-frequency (RF) impairments related to the sub-THz bands, and therefore, cannot be directly used in these frequencies. In particular, sub$\mathrm{THz}$ systems suffer from strong phase impairments due to the poor performance of high-frequency oscillators. We therefore investigate in this paper the optimization of the digital signal processing to mitigate the impact of phase noise (PN) on communication performance within the frame of the highcapacity backhaul.

Optimum receivers for common PN models are listed in [3]. However, these detectors result in complex implementation. Furthermore, optimizing the constellation for PN channels is a widely investigated problem. Stat-of-the art researches have exploited numerical optimization algorithms to design the constellation [4] [5] or to define its parametric shape [6] in order to minimize the symbol error probability or maximize the mutual information. Most of the proposed constellations are efficient but local and unstructured solutions leading to complex binary labelling and demodulation. Conversely, we pursue in this work a pragmatic approach supported by a theoretical framework.

The contributions of this paper are the following. First, we characterize the sub- $\mathrm{THz}$ propagation channels using ray- based deterministic numerical simulations. In addition, we outline the appropriate oscillator PN model for sub- $\mathrm{THz}$ systems. Second, the design of PN robust communications is addressed from both receiver and transmitter perspectives. The optimum symbol detection criterion and the corresponding probabilistic demapper is derived for PN channel upon the maximum likelihood (ML) decision rule. We also propose a PN robust modulation scheme defined upon an efficient and structured constellation, adaptable to any signal-to-noise ratio (SNR) and PN variance. Third, the results of systemlevel simulations are presented, considering a high-capacity backhaul scenario in a North-American city downtown. These simulations are based on ray-tracing channel predictions using accurate LiDAR point cloud and a realistic link budget. It is shown that conventional modulation schemes cannot achieve high data rate communications over sub- $\mathrm{THz}$ channels while the proposed dedicated signal processing enables to maintain high spectral efficiency.

The paper is organized as follows. Sec. II introduces the system model by detailing the sub- $\mathrm{THz}$ propagation channel and the oscillator PN. Sec. III is dedicated to the design of PN robust communications. In Sec. IV, system-level simulations are presented and discussed. Finally, Sec. V concludes the paper and outlines some potential perspectives to this work.

\section{SYSTEM MODEL}

\section{A. Propagation channel}

Sparse propagation channel is a kind of norm in mmWave (millimeter-wave) bands, where the line-of-sight (LoS) directpath often is the most dominant component. Many propagation paths that would bring significant power at the sub$6 \mathrm{GHz}$ frequencies are either attenuated/blocked by obstacles or suffer from the weakness of diffractions. Besides, the specular reflections that occur on flat surfaces large compared to the wavelength and do not cross any obstacle, do still produce multiple propagation paths with significant delays and angles diversity, and possibly cause non-LoS reception. Those characteristics are true in the sub-THz spectrum as well, however slightly accentuated.

Gas absorption and rain attenuation must be two critical factors for long-range sub- $\mathrm{THz}$ links, but their cumulative impact remains below $1 \mathrm{~dB}$ at range $200 \mathrm{~m}$, except for the particular case of strong rainfall rate (greater than $50 \mathrm{~mm} / \mathrm{h}$ ) together with peak water-absorption at $180 \mathrm{GHz}$.

The free-space propagation losses increase with the square of the frequency, therefore in many foreseen applications, the propagation channel degradation will have to be compensated 


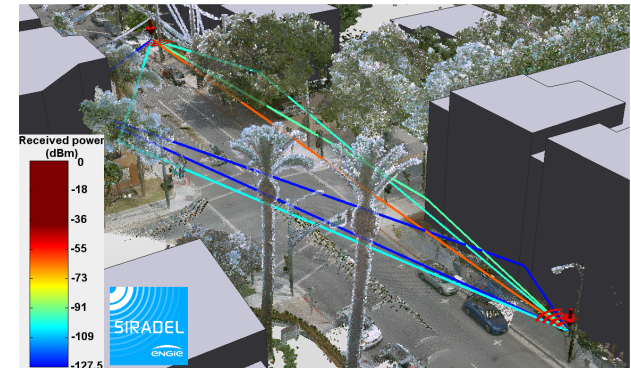

Fig. 1: Propagation paths with ray-tracing and LiDAR data

by high-gain antennas, which comes with high-directivity and antenna alignment constraints.

The propagation channel properties affect both the link-level performance at a given SNR and the service coverage. Thus a fine characterization is required for realistic evaluation of new sub-THz technologies. Today the channel measurements are complex and costly. However only few channel sounding campaigns have been published yet, we can mention those recently realized inside a commercial hall [7], various indoor scenarios [8] [9], a data center [10] or for outdoor-indoor penetration [11]. Numerical simulation is a very convenient solution to complement this characterization, and produce on-demand channel samples. The Volcano ray-based model has been extended up to the sub-THz frequencies [12]. It is employed in the present study to predict the propagation of an in-street backhaul scenario; then the performance of the proposed modulation scheme has been assessed considering this data combined with highly-directive antennas and different PN conditions. Volcano is taking benefit of a light detection and ranging (LiDAR) point cloud collected in the streets of San José, California, USA. This provides a very accurate 3D representation of the environment, thus allows for a very realistic prediction of the blockages and losses due to trees and street furniture as depicted in Fig. 1.

\section{B. Phase noise}

In communication systems, oscillator PN results from the amplification and integration by the phase-locked loop (PLL) of noise sources within the circuitry [13]. Subsequently, PN defines a correlated stochastic process. Under the assumption that the oscillator is only subject to thermal noise, the oscillator PN $\phi$ is described by the superposition of a correlated Wiener (Gaussian random-walk) process and an uncorrelated Gaussian one [13] respectively expressing the integration and amplification of white noise by the PLL. The spectrum of oscillator $\mathrm{PN}$ is in this case described by a colored $K_{2} / f^{2}$ characteristic (Wiener PN) and a white noise floor $K_{0}$ (Gaussian PN). Nevertheless, for high-rate communications, it has been shown in [14] that the influence on communication performance of Wiener PN is negligible compared to the Gaussian one. In other words, the oscillator noise floor amounts to the main contribution of the observed PN for wide-bandwidth systems. These results have been confirmed in [15] and further characterized through the comparison of a correlated PN model to an uncorrelated one. The main result of this study is that the $\mathrm{PN}$ is appropriately modeled by an uncorrelated process if

$$
N \cdot f_{0}^{2} \cdot T^{2} \leq \frac{\ln (2)}{2 \pi^{2}}
$$

where $N$ is the frame length, $f_{0}$ the corner frequency of the oscillator and $T$ is the symbol duration. Let us derive the numerical application for the considered scenario: $1 / T=1$ $\mathrm{GHz}, N=20$ symbols and we may set $f_{0}=1 \mathrm{MHz}$ with regard to the state-of-the-art sub-THz oscillator in [16]. It follows that Eq. (1) is satisfied for these system parameters. For these reasons, it is relevant to describe the impact of PN on received symbols by a zero-mean Gaussian distribution with variance $\sigma_{p}^{2}$. Considering a complex additive white Gaussian noise (AWGN) channel impacted by PN, the received symbol $r$ is hence expressed by

$$
\begin{gathered}
r=s \cdot e^{j \phi}+n, \\
\phi \sim \mathcal{N}\left(0, \sigma_{p}^{2}\right) .
\end{gathered}
$$

where $s$ is the modulated symbol from constellation $\mathcal{C}$ with modulation order $M$ and average energy $E_{s}, \phi$ denotes the PN and $n$ is an additive white Gaussian noise (AWGN) modelling thermal noise. Regarding numerical simulations, we further consider three PN levels: no PN $\sigma_{p}^{2}=0 \mathrm{rad}^{2}$, medium PN $\sigma_{p}^{2}=10^{-2} \operatorname{rad}^{2}$ and strong $\sigma_{p}^{2}=10^{-1} \mathrm{rad}^{2}$. The medium and strong PN levels respectively correspond to a noise floor spectral density $K_{0}$ of $-110 \mathrm{~dB}$ and $-100 \mathrm{~dB}$ for a system bandwidth of $1 \mathrm{GHz}$. Numerical values are obtained using $\sigma_{p}^{2}=K_{0} \cdot B$.

\section{Phase Noise Robust Communications}

\section{A. Receiver algorithm design}

We investigate in this paragraph the design of the optimum receiver for the considered PN channel. For independent and equiprobable symbols, the symbol error probability (SEP) is minimized by the ML detection criterion [17]. The ML decision rule is expressed from the channel likelihood function $p(r \mid s)$ by

$$
\hat{s}=\underset{s \in \mathcal{C}}{\arg \max } p(r \mid s) .
$$

It follows from the system model described in Eq. (2) and from a high-SNR assumption that the channel likelihood function may be efficiently approximated as a bivariate Gaussian - for a detailed derivation see [18]. Denoting the amplitude and phase of symbols respectively with subscripts $\rho$ and $\theta$, the received symbols are distributed as follows:

$$
\begin{aligned}
r_{\rho} & \sim \mathcal{N}\left(s_{\rho}, \sigma_{n}^{2}\right) \\
r_{\theta} & \sim \mathcal{N}\left(s_{\theta}, \sigma_{p}^{2}+\sigma_{n}^{2} / E_{s}\right)
\end{aligned}
$$

Substituting the latter equation into the ML rule, we obtain

$$
\hat{s}=\underset{s \in \mathcal{C}}{\arg \min } d_{\gamma}^{2}(r, s),
$$

defined upon the polar metric

$$
d_{\gamma}^{2}(r, s)=\left(r_{\rho}-s_{\rho}\right)^{2}+\frac{\left(r_{\theta}-s_{\theta}\right)^{2}}{\gamma^{2}},
$$

where $\gamma^{2}=\sigma_{p}^{2} / \sigma_{n}^{2}+1 / E_{s}$. This ML decision rule, hereafter referred to as the polar receiver, is expressed upon a welldefined metric $d_{\gamma}$ and demonstrates a simpler implementation than state-of-the art solutions [5]. It is worth mentioning that in contrast to the Euclidean detector - optimal for the AWGN 
channel, this receiver expresses a joint phase-amplitude detection criterion which adapts its decisions to the $\mathrm{PN}$ variance and to the SNR. Nevertheless, a probabilistic demapper is required when it comes to integrating a forward error correction (FEC) scheme with a soft-input decoding algorithm to improve communication performance. Accordingly, the evaluation of the bit log-likelihood ratio is straightforward from the definition of the polar metric $d_{\gamma}$ and is outlined in [18].

\section{B. Modulation scheme optimization}

In this paragraph, we propose an optimized modulation scheme achieving robustness over PN channels. Constellation design for PN channels is a well-studied problem. State-of-the art solutions use numerical optimization to minimize the SEP [3] or to maximize the mutual information on PN channels. The obtained constellations exhibit significant performance gains in comparison to conventional ones. Nevertheless, the optimized modulations are unstructured and defined for a unique SNR and PN variance, and hence are local solutions leading to complex implementation. Conversely, we introduce in this paper a structured and robust constellation minimizing the SEP with a comprehensive approach for any SNR and PN variance. To do so, it is first required to state that the SEP is expressed upon the minimum polar distance between constellation points i.e. $\min d_{\gamma}\left(s, s^{\prime}\right)$. Subsequently, and similarly to the AWGN channel, designing the constellation that minimizes the SEP is equivalent to characterizing the constellation that maximizes the minimum distance. Under the constraints of fixed modulation order $M$ and $E_{s}$ average energy, this problem may be interpreted as a sphere packing problem in the polar domain, i.e. the phase-amplitude coordinates space. It follows easily that the near-optimal constellation relies on an hexagonal lattice in the polar domain - similarly to the solution for the AWGN channel in the complex plane. However, exploiting a rectangular lattice in the polar domain rather than an hexagonal one leads to a greatly simplified implementation for a negligible performance loss. As a result, we propose the polar quadrature amplitude modulation ( $\mathrm{P}-$ QAM). Its constellation, depicted in Fig. 2, is defined by two parameters $M$ and $\Gamma$ respectively the modulation order and the modulation shape characterized by the number of circles on which modulation points are distributed. The modulation scheme can be adapted to any SNR and PN variance by setting appropriately parameters $M$ and $\Gamma$ to achieve performance gain. By way of illustration, the constellation in Fig. 2a is suited for medium SNR - medium PN channels whereas the one in Fig. $2 b$ is more efficient for a high SNR - strong PN scenario.

\section{System-LEVEl Simulations}

\section{A. Assessment of physical layer performance}

The performance of the physical layer were first assessed to determine the best set of parameters: coding rate, modulation order $M$ and modulation shape $\Gamma$ given the SNR, the PN level and the targeted packet error rate of $10^{-2}$. Concerning the physical layer, we assume a single carrier modulation perfectly synchronized. The channel propagation gain and phase shift are considered perfectly estimated and compensated. A FEC scheme based on the 5GNR LDPC with an input packet size of

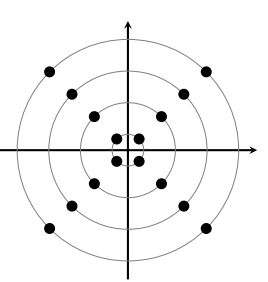

$M=16 \quad \Gamma=4$

(a) medium SNR - medium PN

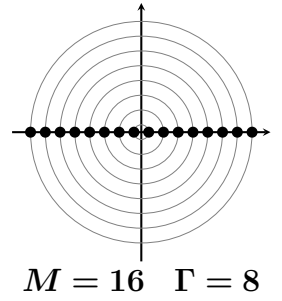

(b) high SNR - strong PN
Fig. 2: Illustration of the proposed modulation: P-QAM

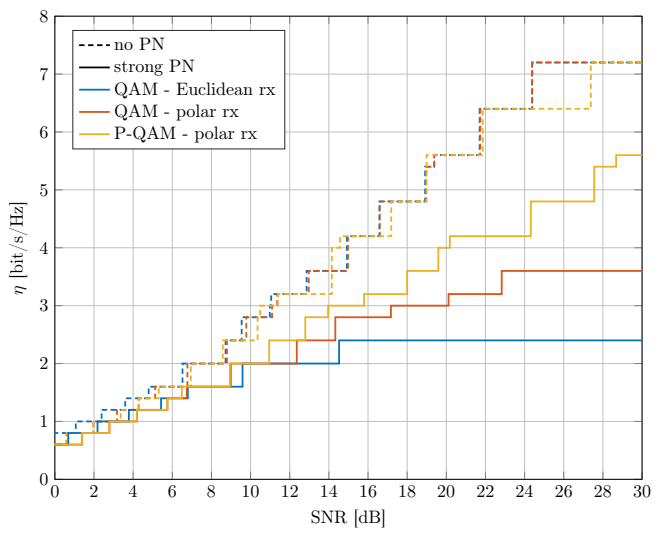

Fig. 3: Highest achievable spectral efficiency given the SNR, the PN level and the modulation/demodulation strategy

1500 bytes is implemented with a coding rate ranging from 0.3 to 0.9 . Fig. 3 present the highest achievable spectral efficiency for two PN levels: no PN and strong PN, and three modulation/demodulation schemes: quadrature amplitude modulation (QAM) with Euclidean receiver, QAM with polar receiver and P-QAM with polar receiver. These results clearly highlight the benefits of using optimized modulation and demodulation schemes especially to target high spectral efficiencies in the presence of PN. It should be mentioned that in low SNR regime and without $\mathrm{PN}$, the polar receiver exhibits a spectral efficiency loss which can be explained by the derivation of the polar receiver from a high-SNR and strong PN assumption.

\section{B. City center scenario}

The performance of the proposed PN robust schemes, the polar receiver and the P-QAM, have been further assessed through system-level simulations. These numerical evaluations assume a city center environment (from the San José, USA,

TABLE I: Simulation parameters

\begin{tabular}{|l|c|c|}
\hline Parameters & Notation & Values \\
\hline \hline Carrier frequency & $f_{c}$ & $150 \mathrm{GHz}$ \\
\hline Channel bandwidth & $B$ & $1 \mathrm{GHz}$ \\
\hline Allocated bandwidth & $B_{a}$ & $0.8 \mathrm{GHz}$ \\
\hline Thermal noise & $N_{0}$ & $-174 \mathrm{dBm} / \mathrm{Hz}$ \\
\hline Noise figure & $N_{f}$ & $10 \mathrm{~dB}$ \\
\hline Transmit power & $P_{\mathrm{Tx}}$ & $30 \mathrm{dBm}$ \\
\hline Tx/Rx antenna gain & $\left(g_{\mathrm{Tx}}, g_{\mathrm{Rx}}\right)$ & $(25,25) \mathrm{dBi}$ \\
\hline Implementation loss & $L$ & $2 \mathrm{~dB}$ \\
\hline Control plane overhead & $\mu$ & $20 \mathrm{p} . \mathrm{c}$ \\
\hline
\end{tabular}




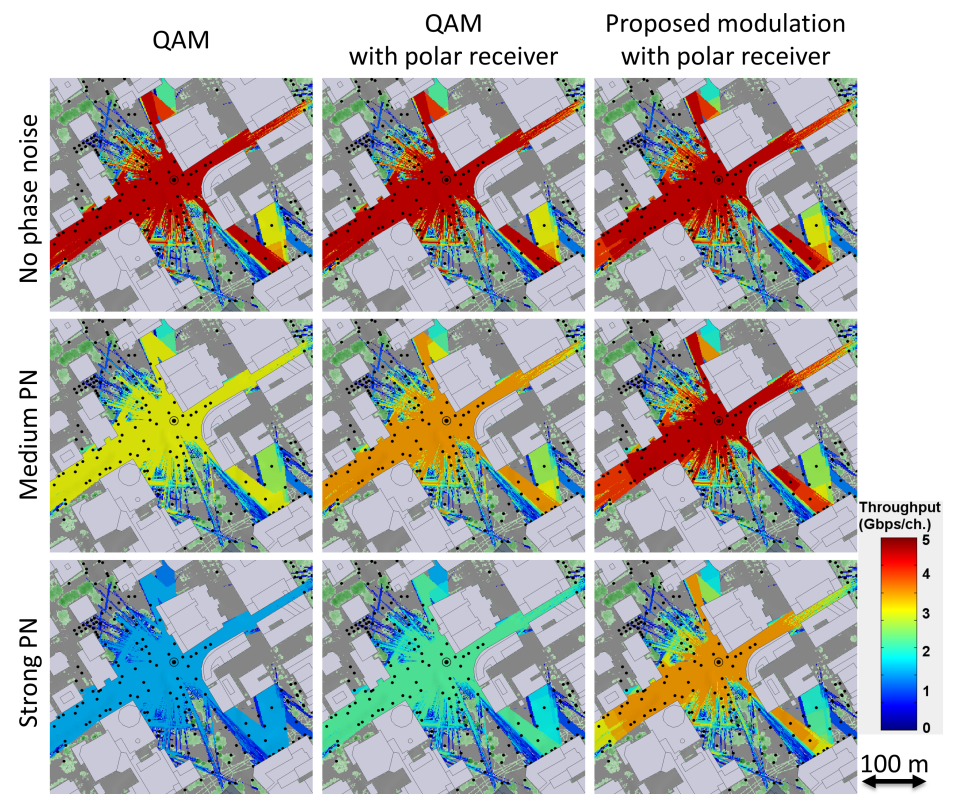

(a) Comparison of standard and proposed solutions when impaired by PN

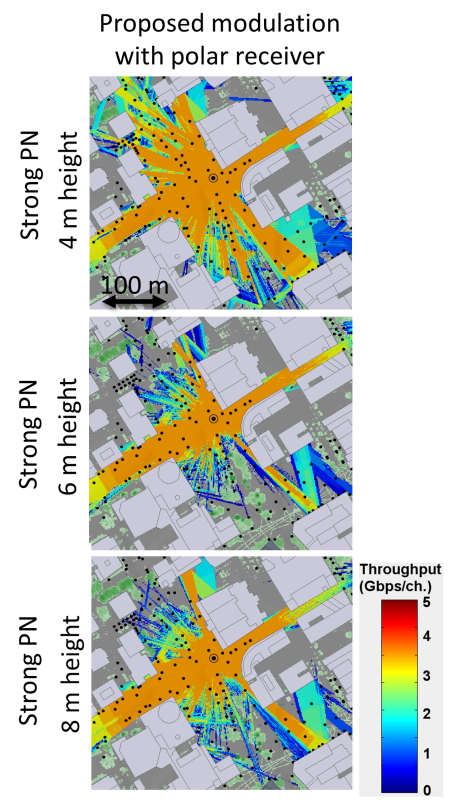

(b) Influence of device height on coverage

Fig. 4: Throughput heat maps for a city center

3D model) while targeting a high capacity mesh backhaul application. The system performance is characterized for an outdoor mesh backhaul situation, which could be exploited to feed an ultra-broadband mobile access network or fixed wireless access (FWA) network. The sub-THz devices are supposed to be installed on lampposts, located along the streets at 8 meters above the ground, in order to transport the data streams from a fiber PoP (Point of Presence) to dense local high-speed access points that either provide mobile or fixed wireless access typically at a lower frequency. The antennas of the sub-THz devices are considered with $6^{\circ}$ beamwidth, $25 \mathrm{dBi}$ maximum gain. The antenna beam is supposed to be perfectly aligned on the dominant propagation path, which is either the direct LoS path, a reflected path, or an attenuated path. Other transmission parameters are given in Table I. The propagation paths and losses are predicted from the ray-tracing tool. The throughput of each calculated link is obtained from the estimated SNR which is mapped to the spectral efficiency of the considered modulation scheme using the previous performance assessment in Sec. IV-A. It should be noticed that the throughput is estimated per channel of 1 $\mathrm{GHz}$ bandwidth. Thereupon, a channel bonding scheme could increase the aggregated throughput and allow to benefit from the available free spectrum offered in the sub-THz bands. The choice of a channelization of $1 \mathrm{GHz}$ is motivated by the current technology of analog-to-digital converters [19].

The heat maps in Fig. 4a illustrates how the lamppost-tolamppost link performs depending on the distance, the propagation condition, the PN level and the selected modulation scheme. The illustrated environment may be characterized as a square in the middle of a dense urban center with medium tree density. The peak throughput per channel is computed between two aligned sub- $\mathrm{THz}$ devices; the first one at the center of the figure; the second one at any pixel of the map. Note that the real lamppost locations, where this second device could precisely be positioned, are shown by black dots. The connectivity is strongly affected by building and foliage obstructions, while line-of-sight propagation and strong clear reflections leads to the best performance. These throughput heat maps demonstrate that optimizing the modulation and demodulation schemes for PN channels results in significant performance gains for future sub-THz systems.

Finally, Fig. 4b illustrates how the ray-based channel simulation and the link-level performance model could be together exploited to optimize the deployment of a sub- $\mathrm{THz}$ mesh network, as different device locations and heights can be tested very easily. In the shown example, the trees have a significant impact; therefore a change in the antenna height can strongly modify the propagation conditions and thus, the connectivity range.

\section{CONCLUSION}

This paper proposes optimized modulation and demodulation schemes that significantly improve the performance, achievable spectral efficiency, of communication systems impaired by oscillator PN. This offers for future sub-THz systems a valuable low-complexity solution to reduce the severe performance degradation caused by PN. The performance of the proposed schemes have been characterized through systemlevel simulations. The numerical simulations modelling a realistic sub-THz mesh deployment scenario in the streets of a North-American city are based on a deterministic prediction of the propagation channel with ray-tracing tools. Coupling raybased channel simulations with link-level performance models will be further exploited to optimize deployments and propose relevant sub-THz network topologies.

\section{ACKNOWLEDGMENT}

The research leading to these results received funding from the French National Research Agency (ANR-17-CE25-0013) within the frame of the project BRAVE. 


\section{REFERENCES}

[1] T. S. Rappaport, Y. Xing, O. Kanhere, S. Ju, A. Madanayake, S. Mandal, A. Alkhateeb, and G. C. Trichopoulos, "Wireless Communications and Applications Above $100 \mathrm{GHz}$ : Opportunities and Challenges for 6G and Beyond," IEEE Access, vol. 7, pp. 78 729-78 757, 2019.

[2] J.-B. Doré, Y. Corre, S. Bicais, J. Palicot, E. Faussurier, D. Kténas, and F. Bader, "Above-90GHz Spectrum and Single-Carrier Waveform as Enablers for Efficient Tbit/s Wireless Communications," in 25th International Conference on Telecommunications (ICT'2018), SaintMalo, France, Jun. 2018.

[3] R. Krishnan, A. G. i Amat, T. Eriksson, and G. Colavolpe, "Constellation optimization in the presence of strong phase noise," IEEE Transactions on Communications, vol. 61, no. 12, pp. 5056-5066, December 2013.

[4] F. Kayhan and G. Montorsi, "Constellation design for channels affected by phase noise," in 2013 IEEE International Conference on Communications (ICC), June 2013, pp. 3154-3158.

[5] R. Krishnan, "On the impact of phase noise in communication systems - performance analysis and algorithms," Ph.D. dissertation, Chalmers University of Technology, 2015.

[6] A. Ugolini, A. Piemontese, and T. Eriksson, "Spiral constellations for phase noise channels," IEEE Transactions on Communications, pp. 1-1, 2019.

[7] S. Nguyen, J. Jarvelainen, A. Karttunen, K. Haneda, and J. Putkonen, "Comparing Radio Propagation Channels Between 28 and $140 \mathrm{GHz}$ Bands in a Shopping Mall," in 12th European Conference on Antennas and Propagation (EuCAP), April 2018.

[8] L. Pometcu and R. D'Errico, "Characterization of Sub-THz and mmWave Propagation Channel for Indoor Scenarios," in 12th European Association on Antennas and Propagation (EurAAP 18), Apr 2018.

[9] L. Pometcu and R. D'Errico, "Channel Model Characteristics in D-Band for NLOS Indoor Scenarios," 2019 13th European Conference on Antennas and Propagation (EuCAP), pp. 1-4, 2019.

[10] M. Eckhardt, T. Doeker, S. Rey, and T. Kürner, "Measurements in a Real Data Center at $300 \mathrm{GHz}$ and Recent Results," in 13rd European Conference on Antennas and Propagation (EuCAP), March 2019.

[11] T. Xing and T. S. Rappaport, "Propagation Measurement System and Approach at $140 \mathrm{GHzMoving}$ to $6 \mathrm{G}$ and Above $100 \mathrm{GHz}$," in 2018 IEEE Global Communications Conference (GLOBECOM), December 2018.

[12] G. Gougeon, Y. Corre, and M. Z. Aslam, "Ray-based Deterministic Channel Modelling for sub-THz Band," in 2019 IEEE International Symposium on Personal, Indoor and Mobile Radio Communications (PIMRC), September 2019.

[13] A. Demir, "Computing Timing Jitter From Phase Noise Spectra for Oscillators and Phase-Locked Loops With White and 1/f Noise," IEEE Transactions on Circuits and Systems I: Regular Papers, vol. 53, no. 9, pp. 1869-1884, Sept 2006.

[14] M. R. Khanzadi, D. Kuylenstierna, A. Panahi, T. Eriksson, and H. Zirath, "Calculation of the performance of communication systems from measured oscillator phase noise," IEEE Transactions on Circuits and Systems I: Regular Papers, vol. 61, no. 5, pp. 1553-1565, May 2014.

[15] S. Bicaïs and J.-B. Doré, "Phase Noise Model Selection for Sub-THz Communications," in 2019 IEEE Global Communication Conference (GLOBECOM), December 2019.

[16] S. Li, D. Fritsche, C. Carta, and F. Ellinger, "A 200-GHz Sub-Harmonic Injection-Locked Oscillator with 0-dBm Output Power and 3.5DCto-RF-Efficiency," in 2018 IEEE Radio Frequency Integrated Circuits Symposium (RFIC), June 2018, pp. 212-215.

[17] J. Proakis, Digital Communications 5th Edition, ser. McGraw-Hill series in electrical and computer engineering : communications and signal processing. McGraw-Hill, 2007.

[18] S. Bicaïs, J.-B. Doré, and J.-L. Gonzalez Jimenez, "On the Optimum Demodulation in the Presence of Gaussian Phase Noise," in 2018 International Conference on Telecommunications (ICT), June 2018.

[19] B. Murmann. "ADC Performance Survey 1997-2019", [Online]. Available: http://web.stanford.edu/ murmann/adcsurvey.html. 